\title{
An Embedding into a Substructure of the r.e. Turing Degrees
}

\author{
Patrizio Cintioli ${ }^{1}$ \\ ${ }^{1}$ Dipartimento di Matematica e Informatica, Università di Camerino, Camerino, Italy \\ Correspondence: Patrizio Cintioli, Dipartimento di Matematica e Informatica, Via Madonna delle Carceri, 9, 62032 \\ Camerino(MC), Italy. E-mail: patrizio.cintioli@unicam.it
}

\author{
Received: September 20, 2015 Accepted: October 30, 2015 Online Published: November 19, 2015 \\ doi:10.5539/jmr.v7n4p137 \\ URL: http://dx.doi.org/10.5539/jmr.v7n4p137
}

\begin{abstract}
Let $\left(I_{m}, \leq\right)$ be the partial ordering of the $m$-introimmune r.e. Turing degrees. We wonder if such structure is an upper semi-lattice. We give a partial answer, by embedding some Boolean algebras into $\left(I_{m}, \leq\right)$.
\end{abstract}

Keywords: recursively enumerable sets, Turing degrees, many-one reducibility, generalized minimal-pair method

\section{Introduction}

\subsection{Introduction to the Problem}

At the end of the sixties some recursion theorists wondered if there were sets of natural numbers without subsets of higher Turing degrees, and if so what would be their degree of unsolvability. This question was solved by Soare, who proved their existence in (Soare, 1969), and by Jockusch (Jockusch, 1973) and Simpson (Simpson 1978), who proved respectively that they cannot be neither arithmetic nor hyperarithmetic. A possible continuation of this study is to consider strong reducibilities $\leq_{r}$ and to see whether or not there are arithmetical sets without subsets of higher $r$-degrees. We recall that a reducibility $\leq_{r}$ is called strong reducibility if it implies the Turing reducibility $\leq_{T}$, that is $\leq_{r}$ is different from $\leq_{T}$ and $A \leq_{r} B \Rightarrow A \leq_{T} B$ for every sets $A, B$ of natural numbers. The unfamiliar reader on these reducibilities can see e.g. (Odifreddi, 1981), as well as a monograph on Computability Theory as, e.g., either (Soare, 1987), or (Odifreddi, 1989). A first positive answer to the above question for the conjunctive reducibiity $\leq_{c}$ is contained in (Cintioli \& Silvestri, 2003). In partuicular, in (Cintioli \& Silvestri, 2003) has been defined the concept of $r$-introimmune set for a given reducibility $\leq_{r}$ : an infinite set $A$ is $r$-introimmune if for every $B \subseteq A$ with $|A-B|=\infty$ it holds that $A \not_{r} B$. So, $r$-introimmune sets have no subsets of higher $r$-degrees for all the reducibilities $\leq_{r}$ studied in computability theory. Until today we know that for all the strong reducibilities $\leq_{r}$ up to the truth-table reducibility $\leq_{t t}$ there are $r$-introimmune arithmetical sets. In particular, there are $t t$-introimmune sets in $\Delta_{2}^{0}$ (Ambos-Spies, 2003) and there are $m$-introimmune sets in $\Pi_{1}^{0}$ (Cintioli, 2005).

Whenever someone discover the existence of sets with a certain property, one possible natural continuation is to understand how they are distributed in some area. In this paper we consider the $m$-introimmune property, and the existence of $m$ introimmune sets in the class $\Pi_{1}^{0}$ suggests to study how they are distributed in the partial ordering $(\mathbf{R}, \leq)$ of the r.e. Turing degrees, which is one of the most important structures studied in computability theory. The natural way to proceed is to collect all the r.e. Turing degrees containing $m$-introimmune sets in the substructure $\mathcal{I}_{m}$ and to study which properties $\mathcal{I}_{m}$ owns. Let us define formally $I_{m}$.

Definition 1.1 Let $I_{m}=\left(I_{m}, \leq_{I_{m}}\right)$, where $I_{m}=\{\mathbf{a} \in \mathbf{R}: \mathbf{a}$ contains a $m$-introimmune set $\}$ and $\leq_{I_{m}}$ is the restriction of $\leq$ to $I_{m}$. From now on we will write simply $\leq$ instead of $\leq_{I_{m}}$.

Since every $m$-introimmune set is immune (Cintioli \& Silvestri, 2003), the Turing degree $\mathbf{0}$ of recursive sets is not in $I_{m}$. We know that $\mathcal{I}_{m}$ has the maximum element $\mathbf{0}^{\prime}$ (Cintioli, 2005), and that $\mathcal{I}_{m}$ has no minimum element (Cintioli, 2014). In this paper we wonder whether or no $\mathcal{I}_{m}$ is an upper semi-lattice.

\subsection{Method}

We know that cohesiveness implies $m$-introimmunity (Cintioli, 2005), and that each $m$-introimmune set is immune (Cintioli \& Silvestri, 2003). Nevertheless, $m$-introimmunity is not hereditary under inclusion. Therefore, we cannot apply the Upward Closure Theorem (Jockusch, 1973) to deduce that $\mathcal{I}_{m}$ is an upper semi-lattice.

However, the Upward Closure Theorem provides a sufficient condition. Let $C$ be the class of all the cohesive r.e. Turing degrees, which is a proper subclass of $I_{m}$. Then, by the Upward Closure Theorem of Jockusch (1973), given any two elements $\mathbf{a}, \mathbf{b} \in I_{m}$ of which at least one in $C$, the least upper bound $\mathbf{a} \oplus \mathbf{b}$ exists in $C \subset I_{m}$. But we do not know if the least upper bound exists in $I_{m}$ in case both $\mathbf{a}$ and $\mathbf{b}$ are not in $C$.

A sufficient condition to establish that $\left(I_{m}, \leq\right)$ is an upper semi-lattice is to prove that given any two elements in $I_{m}-C$ 
their sup is in $I_{m}$. We give here a partial result, by showing that a portion of $\left(I_{m}-C, \leq\right)$ is an upper-semi-lattice. We get this by embedding some uniformly recursive Boolean algebras into $\left(\left(I_{m}-C\right) \cup\{\mathbf{0}\}, \leq\right)$ preserving sups and infs. We preserve infs only for technical reasons, namely, to get one-one maps. We obtain the further results that every countable distributive lattice is embeddable into $\left(\left(I_{m}-C\right) \cup\{\mathbf{0}\}, \leq\right)$ preserving sups and infs, and that every countable partial ordering is embeddable in $\left(I_{m}-C, \leq\right)$. We give in the next section 2 some notations and terminology. In section 3 we prove that the $m$-introimmunity property is not hereditary under inclusion. In section 4 we give our main theorem on the embeddability of some uniformly recursive Boolean algebras in $\left(\left(I_{m}-C\right) \cup\{\mathbf{0}\}, \leq\right)$. We conclude the paper by observing that it is not possible to prove that $\left(I_{m}, \leq\right)$ is an upper semi-lattice by the direct sum of the representatives, that is if $A$ and $B$ are $m$-introimmune and Turing incomparable, then in general it is not true that $A \oplus B$ is $m$-introimmune.

\section{Notations}

For the terminology we refer to the Soare's book (Soare, 1987). Letter $\mathbb{N}$ denotes the set of natural numbers, and all the sets considered in this paper will be subsets of $\mathbb{N}$. For any set $A, \bar{A}=\mathbb{N}-A$, and $\operatorname{deg}(A)$ denotes the Turing degree of the set $A$. With $\langle\cdot, \cdot\rangle: \mathbb{N}^{2} \rightarrow \mathbb{N}$ and $\langle\cdot, \cdot, \cdot\rangle: \mathbb{N}^{3} \rightarrow \mathbb{N}$ we denote two recursive bijections. Given two sets $A$ and $B$, their direct sum is $A \oplus B:=\{\langle x, 0\rangle: x \in A\} \cup\{\langle x, 1\rangle: x \in B\}$. For every nonempty set $I \subseteq \mathbb{N}$ and for every collection $\left\{B_{i}\right\}_{i \in \mathbb{N}}$ of subsets of $\mathbb{N}$, let $\bigoplus_{i \in I} B_{i}=\left\{\langle x, i\rangle: x \in B_{i}\right\}$. We fix an acceptable numbering $\varphi_{0}, \varphi_{1}, \ldots$ of all the Turing computable unary functions. $W_{0}, W_{1}, \ldots$ is the corresponding enumeration of all the recursively enumerable (r.e.) sets. For every $e \in \mathbb{N}$ and every set $X \subseteq \mathbb{N}$, let $\varphi_{e}^{X}$ be the unary function computed by the oracle Turing machine whose gödelian is $e$, with the aid of the oracle $X$. For every numbers $e, s, x$ and for every oracle $X$ we define $\varphi_{e, s}^{X}(x):=\varphi_{e}^{X}(x)$ if there exists $t \leq s$ such that the $e$-th oracle Turing machine on input $x$ with oracle $X$ halts in exactly $t$ steps; in this case we say that $\varphi_{e, s}^{X}(x)$ is defined and we write $\varphi_{e, s}^{X}(x) \downarrow$; we say that $\varphi_{e, s}^{X}(x)$ is undefined otherwise. We recall that the computation of $\varphi_{e, s}^{X}(x)$ asks the oracle $X$ only numbers less than $s$, that is $s$ is an upper bound of the use function $u(X ;, e, x, s)=$ the maximum element asked to $X$ in the computation of $\varphi_{e, s}^{X}(x)$. Finally, given two sets $A, B \subseteq \mathbb{N}, A$ is many-one reducible to $B$, in short $A \leq_{m} B$, if there exists a recursive function $f: \mathbb{N} \rightarrow \mathbb{N}$ such that for every $x \in \mathbb{N}, x \in A$ if and only if $f(x) \in B$.

\section{No hereditary under Inclusion}

We recall that a property $\mathcal{P}$ of infinite sets is hereditary under inclusion if for every infinite set $A$, if $A$ has property $\mathcal{P}$ then each infinite subset of $A$ has property $\mathcal{P}$. In (Jockusch, 1973), Jockusch proved that: "If $\mathcal{P}$ is any property of infinite sets which is hereditary under inclusion and enjoyed by some arithmetical set, then the class of $\mathcal{P}$-degrees is closed upward". So, if the $m$-introimmunity property was hereditary under inclusion, then $\left(I_{m}, \leq\right)$ would be an upper semi-lattice. However, the $m$-introimmunity property is not hereditary under inclusion.

Theorem 3.1 The m-introimmunity property is not hereditary under inclusion.

Proof We construct an r.e. set $A$ with $\bar{A} m$-introimmune and containing an infinite not $m$-introimmune set. We describe the strategy of the construction.

\subsection{Strategy}

It is enough to ensure the existence of an infinite set $B$ such that $B \oplus B \subseteq \bar{A} . B \oplus B$ is not $m$-introimmune because the recursive function $f(\langle x, 0\rangle)=\langle x, 1\rangle$ and $f(\langle x, i\rangle)=\langle x, i\rangle$ for every $i \neq 0$ is an $m$-reduction of $B \oplus B$ to its co-infinite subset $\{\langle x, 1\rangle: x \in B\}$. The set $A$ will be constructed by the finite-extension method with finite injury. At every stage $s \geq 0$ we will define the finite set $A_{s}$. The construction ensures that $A_{s} \subseteq A_{s+1}$ for every stage $s \geq 0$, and $A=\bigcup_{s \geq 0} A_{s}$. It is enough to meet the following requirements, for every $e \geq 0$.

- $P_{3 e}: W_{e}$ infinite $\rightarrow W_{e} \cap A \neq \emptyset$ (immunity of $\bar{A}$ ),

- $N_{3 e+1}:(\exists x \geq e)[\langle x, 0\rangle,\langle x, 1\rangle \in \bar{A}]$ (infinity of $\bar{A}$ and not hereditarily under inclusion),

- $N_{3 e+2}$ : If $\left\{\varphi_{e}(x): \varphi_{e}(x) \neq x \wedge x \in \bar{A}\right\}$ is infinite, then there is a number $u \in \bar{A}$ such that $\varphi_{e}(u) \notin \bar{A}$ (m-introimmunity of $\bar{A})$.

The fulfillment of all the requirements $\left\{N_{3 e+1}\right\}_{e \geq 0}$ guarantees the existence of an infinite set $B$ with $B \oplus B \subseteq \bar{A}$, namely, the set $B=\{x:\langle x, 0\rangle,\langle x, 1\rangle \in \bar{A}\}$.

The fulfillment of all the requirements $\left\{P_{3 e}\right\}_{e \geq 0}$ and $|\bar{A}|=\infty$ guarantees the immunity of $\bar{A}$. Immunity of $\bar{A}$ together with the fulfillment of all the requirements $\left\{N_{3 e+2}\right\}_{e \geq 0}$ imply $\bar{A} m$-introimmune, as stated in the following lemma.

Lemma 3.2 If all the requirements $\left\{N_{3 e+2}\right\}_{e \geq 0}$ are satisfied and $\bar{A}$ is immune, then $\bar{A}$ is m-introimmune.

Proof For the sake of contradiction, let us suppose that $\bar{A}$ is not $m$-introimmune, and let $\varphi_{e}$ be a recursive function that $m$-reduces $\bar{A}$ to some its subset $X$ with $|\bar{A}-X|=\infty$. Then, the set $\left\{\varphi_{e}(x): \varphi_{e}(x) \neq x \wedge x \in \bar{A}\right\}$ is infinite, as stated in the 
following lemma.

Lemma 3.3 (Cintioli, 2011) Let $C$ be immune, and let $C \leq_{m} Y$ via a recursive function $f$, with $Y \subseteq C$ and $|C-Y|=\infty$. Then the set $\{f(x): f(x) \neq x \wedge x \in C\}$ is infinite.

By hypothesis $N_{3 e+2}$ is satisfied; since $\left\{\varphi_{e}(x): \varphi_{e}(x) \neq x \wedge x \in \bar{A}\right\}$ is infinite, it follows that there is an element $u \in \bar{A}$ such that $\varphi_{e}(u) \notin \bar{A}$, hence $\varphi_{e}$ does not $m$-reduce $\bar{A}$ to $X$, which is a contradiction. This concludes the proof of Lemma 3.2.

\subsection{Requirements Requiring Attention and Active Requirements}

The construction of our set $A$ will be by infinitely many stages. At each stage $s$ we try to satisfy one requirement, if possible, among those not yet satisfied at stage $s$. From now on letter $\mathcal{R}$ will denote any requirement. At every stage $s$ there will be a set of requirements requiring attention; we call a requirement $\mathcal{R}_{m}$ active at stage $s$ if $m$ is the minimum index such that $\mathcal{R}_{m}$ requires attention at $s$. Throughout the construction we will use a restraint function $r: \mathbb{N}^{2} \rightarrow \mathbb{N}$. For every negative requirement $N_{q}, r(q, s)=-1$ means that the requirement is not yet satisfied at stage $s$. We will not use the restraint function for any positive requirement $P_{3 e}$, therefore we will pose $r(3 e, s)=-1$ for every $e, s \geq 0$. We now define formally when a requirement requires attention. For every $e, s \geq 0$, we say that

- requirement $P_{3 e}$ requires attention at stage $s+1 \geq 3 e$ if $W_{e, s} \cap A_{s}=\emptyset$ and there is an element $u \in W_{e, s}$ with $u>\max \{r(n, s): n<3 e\}$

- requirement $N_{3 e+1}$ requires attention at stage $s+1 \geq 3 e+1$ if $r(3 e+1, s)=-1$;

- requirement $N_{3 e+2}$ requires attention at stage $s+1 \geq 3 e+2$ if $r(3 e+2, s)=-1$ and there is an element $u \in \overline{A_{s}}$, $u \leq s+1$, such that $\varphi_{e, s}(u) \downarrow \neq u$ and $\varphi_{e, s}(u)>\max \{r(n, s): n<3 e+2\}$.

\subsection{Actions to Fulfill Requirements}

We describe here the actions to fulfill all the requirements.

- To fulfill $P_{3 e}$ we wait for a stage $s+1 \geq 3 e$ at which $P_{3 e}$ is active w.r.t. some $x$, and we enumerate the minimum such $x$ in $A_{s+1}$.

- To fulfill $N_{3 e+1}$ we wait for a stage $s+1 \geq 3 e+1$ at which $N_{3 e+1}$ is active, and restrain in $\overline{A_{s+1}}$ two elements $\langle x, 0\rangle,\langle x, 1\rangle$ with $x \geq e$ by setting $r(3 e, s+1)=\max \{\langle x, 0\rangle,\langle x, 1\rangle\}$.

- To fulfill $N_{3 e+2}$, we wait for a stage $s+1 \geq 3 e+2$ at which $N_{3 e+2}$ is active w.r.t. some $x$. Then we consider the minimum such $x$, enumerate $\varphi_{e, s}(x)$ in $A_{s+1}$ and restrain $x$ in $\overline{A_{s+1}}$ by setting $r(3 e+2, s+1)=x$.

For all the requirements $\mathcal{R}_{m}$ that are not active at stage $s+1$ we set $r(m, s+1)=r(m, s)$.

\subsection{Injured Requirements}

We say that a negative requirement $N_{m}$ is injured at stage $s+1$ if an element $u \leq r(m, s)$ is enumerated in $A_{s+1}$; in this case $r(m, s+1)$ is settled to -1 . For those requirements $N_{m}$ which are not injured at stage $s+1$, we set $r(m, s+1)=r(m, s)$.

\subsection{Construction of $A$}

- Stage 0 . Set $A_{0}=\emptyset$, and set $r(n, 0)=-1$ for every $n \in \mathbb{N}$.

- Stage $s+1>0$. Let $A_{s}$ be the set constructed up to the end of stage $s$. If there are no requirements requiring attention, then set $A_{s+1}:=A_{s}$ and go to the next stage $s+2$. Otherwise let $\mathcal{R}_{n_{0}}$ be the active requirement, and distinguish the following three cases on $n_{0}$ :

1. $n_{0}=3 e$. Pick the minimum $x$ for which $P_{3 e}$ requires attention. Set $A_{s+1}=A_{s} \cup\{x\}$.

2. $n_{0}=3 e+1$. Pick the minimum $x \geq e$ so that $\langle x, 0\rangle$ and $\langle x, 1\rangle$ are both in $\overline{A_{s}}$. Set $r(3 e, s+1)=\max \{\langle x, 0\rangle,\langle x, 1\rangle\}$ and $A_{s+1}=A_{s}$.

3. $n_{0}=3 e+2$. Pick the minimum $x$ for which $N_{3 e+2}$ requires attention. Set $A_{s+1}=A_{s} \cup\left\{\varphi_{e, s}(x)\right\}$ and set $r(3 e+2, s+1)=x$. 


\subsection{Verifications}

The proof that all the requirements require attention at most finitely often does not involve special arguments outside of the finite-extension method with finite injury, so we omit it. We prove only that each $N_{3 e+2}$ is met; the proofs of the other cases are similar.

Lemma 3.4 Each requirement $N_{3 e+2}$ is met.

Proof Let us suppose that each requirement requires attention at most finitely often, and let us suppose that requirements $P_{3 m}$ and $N_{3 m+1}$ are met for every $m \geq 0$. For the sake of contradiction, let us suppose that some $N_{3 e+2}$ is not met, and let $s_{0}$ be the minimum stage after which no requirement of higher priority than $N_{3 e+2}$ requires attention. Let $X$ be a subset of $\bar{A}$ with $|\bar{A}-X|=\infty$ and $\bar{A} \leq_{m} X$ via the recursive function $\varphi_{e}$. It must necessarily be $r\left(3 e+2, s_{0}\right)=-1$, because if it was $r\left(3 e+2, s_{0}\right)=u>0$ then at some stage $s^{\prime}+1<s_{0}$ the number $\varphi_{e, s^{\prime}}(u)$ has been enumerated in $A_{s^{\prime}+1}$ and $N_{3 e+2}$ was not injured up to $s_{0}$. Since $N_{3 e+2}$ will not be injured anymore after $s_{0}$, it would be $u \in \bar{A}$ and $\varphi_{e}(u) \notin \bar{A}$, contrary to the assumption that $\varphi_{e} m$-reduces $\bar{A}$ to $X$. The set $\bar{A}$ is immune because all the requirements $P_{3 e}$ and $N_{3 e+1}$ are met. Thus, by Lemma 3.3 the set $\left\{\varphi_{e}(x): \varphi_{e}(x) \neq x \wedge x \in \bar{A}\right\}$ is infinite. Let $s^{\prime \prime}+1 \geq s_{0}$ be the minimum stage such that there exists $x \leq s^{\prime \prime}+1$ with

- $\varphi_{e, s^{\prime \prime}}(x) \downarrow \neq x$ and

- $\varphi_{e, s^{\prime \prime}}(x)>\max \left\{r\left(n, s^{\prime \prime}\right): n<3 e+2\right\}$.

At stage $s^{\prime \prime}+1 N_{3 e+2}$ requires attention via $x$, and by hypothesis is active. If $x^{\prime}$ is the minimum w.r.t. $N_{3 e+2}$ requires attention, then the number $\varphi_{e, s^{\prime \prime}}\left(x^{\prime}\right)$ is enumerated in $A_{s^{\prime \prime}+1}$ and $x^{\prime}$ is restrained in $\overline{A_{s^{\prime \prime}+1}}$ by $\operatorname{setting} r\left(3 e+2, s^{\prime \prime}+1\right)=x^{\prime}$. This action will not be injured anymore, therefore $x^{\prime} \in \bar{A}$ and $\varphi_{e}\left(x^{\prime}\right) \notin \bar{A}$, contrary to the assumption that $\varphi_{e}$ is a $m$ reduction of $\bar{A}$ to $X$. This concludes the proof of Lemma 3.4.

This concludes the proof of Theorem 3.1 .

\section{Embedding}

We know that every cohesive set is $m$-introimmune (Cintioli, 2005), and that the class of cohesive Turing degrees is upward closed (Jockusch, 1973). Therefore, by taking any two elements $\mathbf{a}, \mathbf{b} \in I_{m}$ of which at least one cohesive, the least upper bound $\mathbf{a} \oplus \mathbf{b}$ is in $I_{m}$. But we do not know if the least upper bound is in $I_{m}$ in case both $\mathbf{a}$ and $\mathbf{b}$ are not cohesive. Let $C$ be the class of all the cohesive r.e. Turing degrees, which is a proper subclass of $I_{m}$. We ask if given any two elements $\mathbf{a}, \mathbf{b} \in I_{m}-C, \mathbf{a} \oplus \mathbf{b}$ is in $I_{m}$. We give here a partial answer by showing that every uniformly recursive Boolean algebra of subsets of $\mathbb{N}$ having $\emptyset$ as minimum is lattice embeddable in $\left(\left(I_{m}-C\right) \cup\{\boldsymbol{0}\}, \leq\right)$. We will obtain also that every countable distributive lattice is embeddable into $\left(\left(I_{m}-C\right) \cup\{\mathbf{0}\}, \leq\right)$ preserving sups and infs, and that every countable partial ordering is embeddable in $\left(I_{m}-C, \leq\right)$. Let us first fix some terminology. We recall that a lattice $\mathcal{L}=\left(\left\{L_{i}\right\}_{i \in \mathbb{N}}, \subseteq, \cup, \cap\right)$ of subsets of $\mathbb{N}$ is uniformly recursive if the set $\left\{\langle x, i\rangle: x \in L_{i}\right\}$ is recursive. Given a partial ordering $\left(P, \leq_{P}\right)$ and given a one-one map $f: P \rightarrow I_{m}$ we say that:

- $f$ is an embedding of $\left(P, \leq_{P}\right)$ into $\left(I_{m}, \leq\right)$ if $x \leq_{P} y \Leftrightarrow f(x) \leq f(y)$ for every $x, y \in P$;

- if $f$ is an embedding of $\left(P, \leq_{P}\right)$ into $\left(I_{m}, \leq\right)$ and $f$ preserves sups and infs, that is for every $x, y \in P, f(\{\operatorname{in} f\{x, y\}\})=$ inf $\{f(x), f(y)\}$ and $f(\{\sup \{x, y\}\})=\sup \{f(x), f(y)\}$, then $f$ is a lattice embedding of $\left(P, \leq_{P}\right)$ into $\left(I_{m}, \leq\right)$.

The strategy to prove that the uniformly recursive Boolean algebras above are lattice embeddable in $\left(\left(I_{m}-C\right) \cup\{\mathbf{0}\}, \leq\right)$ is to construct a collection $\left\{B_{i}\right\}_{i \in \mathbb{N}}$ of infinite subsets of $\mathbb{N}$ in such a way that $\bigoplus_{i \in \mathbb{N}} B_{i}$ is $m$-introimmune, co-r.e. and low $_{1}$. The co-r.e. property together with the lowness property guarantee that $\bigoplus_{i \in I} B_{i}$ is co-r.e. and not cohesive for every nonempty recursive set $I$. Then, the $m$-introimmunity of $\bigoplus_{i \in \mathbb{N}} B_{i}$ implies the $m$-introimmunity of $\bigoplus_{i \in I} B_{i}$ for every nonempty recursive set $I$. We prove the latter in the following lemma.

Lemma 4.1 Let $\bigoplus_{i \in \mathbb{N}} A_{i}$ be m-introimmune, and let $I \subseteq \mathbb{N}$ be any recursive set such that $\bigoplus_{i \in I} A_{i}$ is infinite. Then $\bigoplus_{i \in I} A_{i}$ is m-introimmune.

Proof For the sake of contradiction, let us suppose that $\bigoplus_{i \in I} A_{i}$ is not $m$-introimmune, and let $X \subseteq \bigoplus_{i \in I} A_{i}$ such that $\left(\bigoplus_{i \in I} A_{i}\right) \leq_{m} X$ via $g$, with

$$
\left|\left(\bigoplus_{i \in I} A_{i}\right)-X\right|=\infty
$$


in particular $X \cap\left(\bigoplus_{i \in \mathbb{N}-I} A_{i}\right)=\emptyset$. We get a contradiction by proving that $\bigoplus_{i \in \mathbb{N}} A_{i}$ is not $m$-introimmune. Let us define $Y=X \cup\left(\bigoplus_{i \in \mathbb{N}-I} A_{i}\right)$. Then, $Y \subseteq \bigoplus_{i \in \mathbb{N}} A_{i}$ and

$$
\left|\left(\bigoplus_{i \in \mathbb{N}} A_{i}\right)-Y\right|=\left|\left(\bigoplus_{i \in I} A_{i}\right)-X\right|=\infty .
$$

Let $b_{0} \notin Y$ be fixed. Then, $\left(\bigoplus_{i \in \mathbb{N}} A_{i}\right) \leq_{m} Y$ via $f$ defined in the following way:

a) for every $i \in I, f(\langle x, i\rangle)=g(\langle x, i\rangle)$, if $g(\langle x, i\rangle)=\langle y, j\rangle$ for some $j \in I ; f(\langle x, i\rangle)=b_{0}$ otherwise;

b) for every $i \notin I, f(\langle x, i\rangle)=\langle x, i\rangle$.

Given any $\langle x, k\rangle \in \mathbb{N}$ let us prove that

$$
\langle x, k\rangle \in \bigoplus_{i \in \mathbb{N}} A_{i} \Leftrightarrow f(\langle x, k\rangle) \in Y .
$$

- $(\Rightarrow)$ Let us suppose that $\langle x, k\rangle \in \bigoplus_{i \in \mathbb{N}} A_{i}$; we have two cases: $k \in I$ and $k \notin I$.

1. If $k \in I$, then $f(\langle x, k\rangle)=g(\langle x, k\rangle) \in X \subseteq Y$.

2. If $k \notin I$, then

$$
\langle x, k\rangle \in \bigoplus_{i \in \mathbb{N}} A_{i} \Rightarrow\langle x, k\rangle \in \bigoplus_{i \in \mathbb{N}-I} A_{i}
$$

so $f(\langle x, k\rangle)=\langle x, k\rangle \in \bigoplus_{i \in \mathbb{N}-I} A_{i} \subseteq Y$.

- $(\Leftarrow)$ Let us suppose that $\langle x, k\rangle \notin \bigoplus_{i \in \mathbb{N}} A_{i}$, from which $g(\langle x, k\rangle) \notin X$. Again, we have two cases: $k \in I$ and $k \notin I$.

1. $k \in I$. In this case we have two subcases (a) and (b):

(a) $g(\langle x, k\rangle)=\langle y, j\rangle$ with $j \in I$. In this subcase $f(\langle x, k\rangle)=g(\langle x, k\rangle)$. But $g(\langle x, k\rangle) \notin X$, hence $f(\langle x, k\rangle) \notin Y$.

(b) $g(\langle x, k\rangle)=\langle y, j\rangle$ with $j \notin I$. In this subcase $f(\langle x, k\rangle)=b_{0} \notin Y$.

2. If $k \notin I$, then $f(\langle x, k\rangle)=\langle x, k\rangle \notin \bigoplus_{i \in \mathbb{N}} A_{i}$ by hypothesis, thus $f(\langle x, k\rangle) \notin Y$.

Now, we prove our main theorem.

Theorem 4.2 Let $\mathcal{B}=\left(\left\{\alpha_{i}\right\}_{i \in \mathbb{N}}, \subseteq, \cup, \cap\right)$ be any uniformly recursive Boolean algebra of subsets of $\mathbb{N}$ containing the minimum element $\emptyset$. Then $\mathcal{B}$ is lattice embeddable in $\left(\left(I_{m}-C\right) \cup\{\mathbf{0}\}, \leq\right)$.

Proof We show that there is a one-one map $f:\left\{\alpha_{i}\right\}_{i \in \mathbb{N}} \rightarrow\left(I_{m}-C\right) \cup\{\mathbf{0}\}$ such that for every $\alpha_{i}, \alpha_{j}$,
(a) $\alpha_{i} \subseteq \alpha_{j} \Leftrightarrow f\left(\alpha_{i}\right) \leq f\left(\alpha_{j}\right)$,
(b) $f\left(\alpha_{i} \cup \alpha_{j}\right)=\sup \left\{f\left(\alpha_{i}\right), f\left(\alpha_{j}\right)\right\}$,
(c) $f\left(\alpha_{i} \cap \alpha_{j}\right)=\inf \left\{f\left(\alpha_{i}\right), f\left(\alpha_{j}\right)\right\}$

The strategy of the proof is the following.

\subsection{Strategy}

We construct a collection $\left\{B_{i}\right\}_{i \in \mathbb{N}}$ of infinite sets such that the set $\bigoplus_{i \in \mathbb{N}} B_{i}$ will have the following properties: is low ${ }_{1}$, co-r.e., $m$-introimmune, and for every $\alpha_{i}, \alpha_{j}$ of $\mathcal{B}$, for every set $D$,

$$
\text { if } \begin{aligned}
D \leq_{T}\left(\bigoplus_{k \in \alpha_{i}} B_{k}\right) \text { and } D \leq_{T}\left(\bigoplus_{k \in \alpha_{j}} B_{k}\right) \text {, then } \\
\text { then } D \leq_{T}\left(\bigoplus_{k \in \alpha_{i} \cap \alpha_{j}} B_{k}\right) .
\end{aligned}
$$


Then, we define the map $f$ as

$$
f(\alpha)=\operatorname{deg}\left(\bigoplus_{k \in \alpha} B_{k}\right)
$$

for every $\alpha$ of $\mathcal{B}$. We have to show that $f$ is the desired function satisfying all the conditions (a), (b) and (c).

Claim 4.3f: $\left\{\alpha_{i}\right\}_{i \in \mathbb{N}} \rightarrow\left(I_{m}-C\right) \cup\{0\}$.

Proof From the fact that $\bigoplus_{i \in \mathbb{N}} B_{i}$ is low 1 and co-r.e. it follows that $\bigoplus_{i \in \mathbb{N}} B_{i}$ is not cohesive, because a cohesive co-r.e.

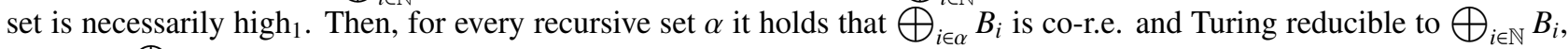
therefore $\bigoplus_{i \in \alpha} B_{i}$ is low 1 , hence not cohesive. Moreover, by Lemma 4.1 it holds that for every recursive nonempty set $\alpha$, $\bigoplus_{i \in \alpha} B_{i}$ is $m$-introimmune. In conclusion, for every nonempty recursive set $\alpha$ the set $\bigoplus_{i \in \alpha} B_{i}$ is not cohesive, co-r.e. an $m$-introimmune, therefore its Turing degree $\operatorname{deg}\left(\bigoplus_{i \in \alpha} B_{i}\right)=f(\alpha)$ is in $I_{m}-C$. Of course, if $\alpha=\emptyset$ then $f(\alpha)=\mathbf{0}$. This concludes the proof of the claim.

Claim $4.4 f$ is one-one.

Proof It is enough to prove that $\alpha_{m} \neq \alpha_{n}$ implies $\left(\bigoplus_{i \in \alpha_{m}} B_{i}\right) \neq_{T}\left(\bigoplus_{i \in \alpha_{n}} B_{i}\right)$, which is true by the following known argument. Without loss of generality, let us suppose that $\alpha_{m} \nsubseteq \alpha_{n}$, and for the sake of contradiction let us assume that

$$
\left(\bigoplus_{k \in \alpha_{m}} B_{k}\right) \leq_{T}\left(\bigoplus_{k \in \alpha_{n}} B_{k}\right) \text {. }
$$

Let $h \in \alpha_{m}-\alpha_{n}$; then, $B_{h} \leq_{T}\left(\bigoplus_{i \in \alpha_{m}} B_{i}\right)$ and by hypothesis $\left(\bigoplus_{i \in \alpha_{m}} B_{i}\right) \leq_{T}\left(\bigoplus_{i \in \alpha_{n}} B_{i}\right)$, so $B_{h} \leq_{T}\left(\bigoplus_{i \in \alpha_{n}} B_{i}\right)$. Moreover, $B_{h} \leq_{T}\left(\bigoplus_{i \in \bar{\alpha}_{n}} B_{i}\right)$. Therefore, by (5) and (6)

$$
B_{h} \leq_{T}\left(\bigoplus_{i \in \alpha_{n} \cap \overline{\alpha_{n}}} B_{i}\right)=\emptyset,
$$

which implies $B_{h}$ recursive. But by Lemma 4.1 for $I=\{h\}$ it follows that $\bigoplus_{i \in\{h\}} B_{i}$ is $m$-introimmune, which implies $B_{h}$ $m$-introimmune, hence nonrecursive, a contradiction. This concludes the proof of the claim.

Claim 4.5 Conditions (a), (b) and (c) hold.

Proof For the condition (a), $(\Rightarrow)$ is obvious, while the argument of the proof of Claim 4.4 proves $(\Leftarrow)$ ). Condition (b) is obvious. Condition (c) follows form ( 5), (6) and by the obvious facts that

$$
\left(\bigoplus_{k \in \alpha_{i} \cap \alpha_{j}} B_{k}\right) \leq_{T}\left(\bigoplus_{k \in \alpha_{i}} B_{k}\right)
$$

and

$$
\left(\bigoplus_{k \in \alpha_{i} \cap \alpha_{j}} B_{k}\right) \leq_{T}\left(\bigoplus_{k \in \alpha_{j}} B_{k}\right) .
$$

This concludes the proof of the claim.

We now describe the construction of the collection $\left\{B_{i}\right\}_{i \in \mathbb{N}}$. We will construct an infinite and co-infinite low 1 r.e. set $A$ in such a way that $\bar{A}=\bigoplus_{i \in \mathbb{N}} B_{i}$, with $\bar{A} m$-introimmune. The construction is by infinitely many stages; at every stage $s$ we will define the finite set $A_{s}$, with $A_{0}=\emptyset$ and $A_{s} \subseteq A_{s+1}$ for every $s \geq 0$. The final set is $A=\bigcup_{s \geq 0} A_{s}$. For every $i, s \in \mathbb{N}$, let

$$
B_{i, s}=\left\{x:\langle x, i\rangle \in \overline{A_{s}}\right\} .
$$

Then, for every $i, s \in \mathbb{N}$ is

$$
\overline{A_{s}}=\bigoplus_{i \in \mathbb{N}} B_{i, s},
$$

with $B_{i, s} \supseteq B_{i, s+1}$ and $\bar{A}=\bigoplus_{i \in \mathbb{N}} B_{i}$, where $B_{i}=\lim _{s \rightarrow \infty} B_{i, s}$. To ensure that the set $\bar{A}=\bigoplus_{i \in \mathbb{N}} B_{i}$ will have the required properties, it suffices to construct $A$ in order to satisfy the following requirements for every $e, m, n, i \in \mathbb{N}$. 


\subsection{Requirements}

For every natural numbers $e, i, m, n \geq 0$ we define the following requirements:

- $P_{5 e}:\left|W_{e}\right|=\infty \rightarrow W_{e} \cap A \neq \emptyset$ (immunity of $\bar{A}$ ),

- $N_{5 e+1}:$ if $\left\{\varphi_{e}(x): \varphi_{e}(x) \neq x \wedge x \in \bar{A}\right\}$ is infinite, then there is an element $x \in \bar{A}$ with $\varphi_{e}(x) \notin \bar{A}$ (m-introimmunity of $\bar{A})$

- $N_{5 e+2}:\left[\left(\exists^{\infty} s\right)\left(\varphi_{e, s}^{A_{s}}(e)\right.\right.$ is defined) $] \Rightarrow \varphi_{e}^{A}(e)$ is defined (lowness of $\left.A\right)$,

- $N_{5\langle i, e\rangle+3}:(\exists x)[x \geq e \wedge\langle x, i\rangle \in \bar{A}]$ (infinity of $\left.B_{i}\right)$,

- $N_{5\langle e, m, n\rangle+4}:\left(\varphi_{e}^{\bigoplus_{i \epsilon \alpha_{m}} B_{i}}=\varphi_{e}^{\bigoplus_{i \in \alpha_{n}} B_{i}}=D\right) \Rightarrow D \leq_{T} \bigoplus_{i \in \alpha_{m} \cap \alpha_{n}} B_{i}$ (preserving infs).

We show that all the above requirements imply all the requested properties for $\bar{A}=\bigoplus_{i \in \mathbb{N}} B_{i}$.

Lemma 4.6 Requirements $P_{5 e}, N_{5\langle i, e\rangle+3}$ and $N_{5\langle i, e\rangle+3}$ guarantee that $\bar{A}$ is m-introimmune.

The proof of this lemma is essentially the same of that of Lemma 3.2, so we omit it.

Lemma 4.7 Requirements $N_{5 e+2}$ guarantee that $A$ is $l_{0} w_{1}$.

Proof The statement follows from the known proposition.

Lemma 4.8 If $E=\bigcup_{t \geq 0} E_{t}$ is a recursively enumerable set and for every $n \in N$

$$
\left[\left(\exists^{\infty} t\right) \varphi_{n, t}^{E_{t}}(n) \downarrow\right] \Rightarrow \varphi_{n}^{E}(n) \text { is defined, }
$$

then $E$ is $\operatorname{low}_{1}$.

Lemma 4.9 Requirements $N_{5\langle e, m, n\rangle+4}$ guarantee (5) and (6).

Proof The natural requirement to get (5) and (6) is

$$
N_{5\langle a, b, m, n\rangle+4}:\left(\varphi_{a}^{\bigoplus_{i \in \alpha_{m}} B_{i}}=\varphi_{b}^{\bigoplus_{i \in \alpha_{n}} B_{i}}=D\right) \Rightarrow D \leq_{T} \bigoplus_{i \in \alpha_{m} \cap \alpha_{n}} B_{i} .
$$

However, under the hypothesis that all the requirements $P_{5 e}, N_{5 e+1}, N_{5\langle i, e\rangle+3}$ and $N_{5\langle e, m, n\rangle+4}$ are satisfied, it follows that all the requirements (7) are satisfied. The proof is an argument due to Posner (see Soare, (1987), page 153). For every $\alpha_{m}, \alpha_{n} \neq \emptyset$, both the sets $\bigoplus_{i \in \alpha_{m}} B_{i}$ and $\bigoplus_{i \in \alpha_{n}} B_{i}$ are not recursive. Since by hypothesis all the requirements $N_{5\langle e, m, n\rangle+4}$ are satisfied, it follows that $\bigoplus_{i \in \alpha_{m}} B_{i} \neq \bigoplus_{i \in \alpha_{n}} B_{i}$. Without loss of generality assume that

$$
\left(\bigoplus_{i \in \alpha_{m}} B_{i}\right) \nsubseteq\left(\bigoplus_{i \in \alpha_{n}} B_{i}\right),
$$

and let

$$
x_{0} \in\left(\bigoplus_{i \in \alpha_{m}} B_{i}\right)-\left(\bigoplus_{i \in \alpha_{n}} B_{i}\right) .
$$

For the sake of contradiction, assume that there are $i_{0}$ and $j_{0}$ such that

$$
\varphi_{i_{0}}^{\bigoplus_{i \in \alpha_{m}} B_{i}}=\varphi_{j_{0}}^{\bigoplus_{i \in \alpha_{n}} B_{i}}=D \text { with } D \not_{T} \bigoplus_{i \in \alpha_{m} \cap \alpha_{n}} B_{i} .
$$

Let $e=e\left(x_{0}, i_{0}, j_{0}\right)$ be such that for every oracle $X$ and every $x \in N$

$$
\varphi_{e}^{X}(x)= \begin{cases}\varphi_{i_{o}}^{X}(x) & \text { if } x_{0} \in X, \\ \varphi_{j_{o}}^{X}(x) & \text { if } x_{0} \notin X .\end{cases}
$$

Then clearly $\varphi_{e}^{\bigoplus_{i \in \alpha_{m}} B_{i}}=\varphi_{i_{0}}^{\bigoplus_{i \epsilon \alpha_{m}} B_{i}}$ and $\varphi_{e}^{\bigoplus_{i \in \alpha_{n}} B_{i}}=\varphi_{j_{0}}^{\bigoplus_{i \in \alpha_{m}} B_{i}}$. It follows from (8) that $\varphi_{e}^{\bigoplus_{i \epsilon \alpha_{m}} B_{i}}=\varphi_{e}^{\bigoplus_{i \in \alpha_{n}} B_{i}}=D$ with $D \mathbb{\leq}_{T}$ $\bigoplus_{i \in \alpha_{m} \cap \alpha_{n}} B_{i}$, contrary to the hypothesis that $N_{5\langle e, m, n\rangle+4}$ is met. 


\subsection{Requirements Requiring Attention and Active Requirements}

In this section we formulate the conditions under which a requirement requires attention. Similarly to the previous Theorem 3.1, we use a restraint function $r: \mathbb{N}^{2} \rightarrow \mathbb{N}$. For every negative requirement $N_{q}$ with $q \neq 5 n+4$ for every $n$, $r(q, s)=-1$ means that the requirement is not yet satisfied at the stage $s$. We do not need the use of the restraint function for the positive requirements $P_{5 e}$, therefore we will pose $r(5 e, s)=-1$ for every $e, s \geq 0$. For every $e, i \geq 0$,

- $P_{5 e}$ requires attention at stage $s+1 \geq 5 e$ if $W_{e, s} \cap A_{s}=\emptyset$ and there exists $x \in W_{e, s}$ such that

$$
x>\max \{r(n, s): n<5 e\}
$$

- $N_{5 e+1}$ requires attention at stage $s+1 \geq 5 e+1$ if $r(5 e+1, s)=-1$ and there exists $x \in \bar{A}_{s}, x \leq s+1$, with $\varphi_{e, s}(x) \downarrow \neq x$ and

$$
\varphi_{e, s}(x)>\max \{r(n, s): n<5 e+1\}
$$

- $N_{5 e+2}$ requires attention at stage $s+1 \geq 5 e+2$ if $\varphi_{e, s}^{A_{s}}(e) \downarrow$ and $r(5 e+2, s)=-1$;

- $N_{5\langle i, e\rangle+3}$ requires attention at stage $s+1 \geq 5\langle i, e\rangle+3$ if $r(5\langle i, e\rangle+3, s)=-1$.

We say that a requirement $\mathcal{R}_{m}$ above is active at stage $s+1$ if $m$ is the minimum for which $\mathcal{R}_{m}$ requires attention at stage $s+1$.

\subsection{Actions to Fulfill Requirements}

We describe here the actions to fulfill requirements. Let $e, i \geq 0$.

- For $P_{5 e}$ : we wait for a stage $s+1 \geq 5 e$ at which it is active w.r.t. to some $x$; then we enumerate the minimum such $x$ in $A_{s+1}$.

- For $N_{5 e+1}$ : we wait for a stage $s+1 \geq 5 e+1$ at which it is active w.r.t. some $x$. Then we consider the minimum such $x$, enumerate $\varphi_{e, s}(x)$ in $A_{s+1}$ and restrain $x$ in $\overline{A_{s+1}}$ by setting $r(5 e+1, s+1)=x$.

- For $N_{5 e+2}$ : we employ the classical method of to obtain a low 1 set, namely, we wait for a stage $s+1 \geq 5 e+2$ such that it is is active. Then we preserve the computation $\varphi_{e, s}^{A_{s}}(e)$ by restraining all the queries made to the oracle by setting $r(5 e+2, s+1)=s$.

- For $N_{5\langle i, e\rangle+3}$ : we wait for a stage $s+1 \geq 5\langle i, e\rangle+3$ at which it is active and we restrain $\langle x, i\rangle$ in $\overline{A_{s+1}}$ by setting $r(5\langle i, e\rangle+3, s+1)=\langle x, i\rangle$, where $x$ is the minimum number such that $\langle x, i\rangle \in \overline{A_{s}}$ and $x \geq e$.

- For $N_{5\langle e, m, n\rangle+4}$ : we use the generalization of the Yates' minimal-pair method due to Thomason (Thomason, 1971), Lerman and Lachlan (see Soare, (1987), page 157 et seq.). At every stage $s$ of the construction of $A$ we define the length function $l(\langle e, m, n\rangle, s)$ for every $e, m, n \in \mathbb{N}$ as:

$$
l(\langle e, m, n\rangle, s)=\max \left\{x:(\forall y<x)\left[\varphi_{e, s}^{\bigoplus_{i \in \alpha_{m}} B_{i, s}}(y) \downarrow=\varphi_{e, s}^{\bigoplus_{i \in \alpha_{n}} B_{i, s}}(y) \downarrow\right]\right\}
$$

Then, at every stage $s \geq 0$ we define the restraint function $r(5\langle e, m, n\rangle+4, s)$ by induction on $\langle e, m, n\rangle$ according to the minimal-pair method, where we recall that the number $s$ is an upper bound of the use function.

Definition 4.10 (Definition of the restraint function $r(\cdot, \cdot)$ ) For every stage $s \geq 0$, let us define

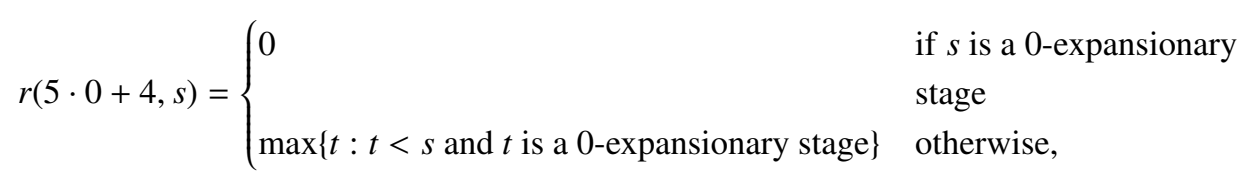

where a stage $s$ is 0 -expansionary if either $s=0$ or

$$
l(0, s)>\max \{l(0, t): t<s\} .
$$

Given $r(5\langle e, m, n\rangle+4, s)$, define $r(5(\langle e, m, n\rangle+1)+4, s)$ as the maximum of:

(1) $r(5\langle e, m, n\rangle+4, s)$, 
(2) $\{t: t<s$ and $r(5\langle e, m, n\rangle+4, t)<r(5\langle e, m, n\rangle+4, s)\}$,

(3) $\{t: t\langle s, r(5\langle e, m, n\rangle+4, t)=r(5\langle e, m, n\rangle+4, s)$ and stage $t$ is $(\langle e, m, n\rangle+1)$-expansionary, if $s$ is not an $(\langle e, m, n\rangle+1)$-expansionary stage $\}$,

where a stage $s$ is $(\langle e, m, n\rangle+1)$-expansionary if either $s=0$ or

$$
(\forall t<s)[r(5\langle e, m, n\rangle+4, t)=r(5\langle e, m, n\rangle+4, s) \Rightarrow l(\langle e, m, n\rangle+1, t)<l(\langle e, m, n\rangle+1, s)] .
$$

It is known that for every $e, m, n \in \mathbb{N}$,

$$
\lim \inf _{s \rightarrow \infty} r(5\langle e, m, n\rangle+4, s)<\infty
$$

(see for example either Soare, (1987), cf. Lemma 1, page 155, or Odifreddi, (1999), page 545).

If $\varphi_{e}^{\bigoplus_{i \epsilon \alpha_{m}} B_{i}}=\varphi_{e}^{\bigoplus_{i \epsilon \alpha_{n}} B_{i}}=D$, then the Thomason's method ensures that $D$ can be decided with the aid of the oracle $\bigoplus_{i \in \alpha_{m} \cap \alpha_{n}} B_{i}$.

\subsection{Injured Requirements}

A negative requirement $N_{p}$ is injured at stage $s+1$ if an element $u \leq r(p, s)$ is enumerated in $A_{s+1}$. At every stage $s+1$, for every $p$ with $p \neq 5 q+4$ for every $q$, we set $r(p, s+1)=-1$ for each injured requirement, while for each not injured requirement $N_{p}$ we set $r(p, s+1)=r(p, s)$. The reason for the condition $p \neq 5 q+4$ is that we should not change the particular definition of the restraint function for the negative requirement $N_{5 q+4}$.

\subsection{Construction of the Set A}

The construction of $A$ is by infinitely many stages. At every stage $s$ we check if there are some requirements requiring attention, and if so we satify that one with the highest priority, that is the active requirement.

- Stage 0. $A_{0}=\emptyset$. Set $r(q, s)=-1$ for every $q, s \geq 0$ with $q \neq 5 n+4$ for every $n \in \mathbb{N}$.

- Stage $s+1$. Let $A_{s}$ be given. If there are no requirements requiring attention, then do nothing, that is set $A_{s+1}=A_{s}$. Otherwise, let $\mathcal{R}_{p}$ be the active requirement, and distinguish the following four cases on $p$ :

- $p=5 e$. Let $x$ be the minimum element for which $P_{5 e}$ requires attention, and set $A_{s+1}=A_{s} \cup\{x\}$.

$-p=5 e+1$. Let $x$ be the minimum for which $N_{5 e+1}$ requires attention. Set $A_{s+1}=A_{s} \cup\left\{\varphi_{e, s}(x)\right\}$ and $r(5 e+1, s+1)=x$.

- $p=5 e+2$. Set $r(5 e+2, s+1)=s$ and $A_{s+1}=A_{s}$.

- $p=5\langle i, e\rangle+3$. Let $x$ be the minimum such that $\langle x, i\rangle \in \overline{A_{s}}$, and $x \geq e$. Set $r(5\langle i, e\rangle+3, s+1)=\langle x, i\rangle$ and $A_{s+1}=A_{s}$.

\section{End construction of set $A$.}

\subsection{Verifications}

The proof that every requirement $\mathcal{R}_{m}$ with $m \neq 5 q+4$ for any $q \geq 0$ requires attention at most finitely often does not involve particular arguments outside of the finite-extension method, so we omit it.

Corollary 4.11 For every $n \neq 5 q+4$ for any $q \geq 0, \lim _{s \rightarrow \infty} r(n, s)$ exists and is finite.

By Corollary 4.11 and (10) it follows

Corollary 4.12 For every $n \geq 0, \liminf _{s \rightarrow \infty} \max \{r(t, s): t \leq n\}<\infty$.

Now it is possible to prove that every requirement is meet.

Lemma 4.13 Every requirement is met.

Proof We prove only the cases $N_{5 e+1}$ and $N_{5\langle e, m, n\rangle+4}$, because for the other cases the proofs are similar to that for $N_{5 e+1}$. So, let us assume that all the requirements $P_{5 e}$ and $N_{5\langle i, e\rangle+3}$ are met for every $e, i \geq 0$. 
- For $N_{5 e+1}$, the proof is very similar to that of the previous Lemma 3.4. Let $s_{0}$ be the minimum stage such that for every $s \geq s_{0}$ no requirement of higher priority than $N_{5 e+1}$ requires attention at stage $s$. For the sake of contradiction, let us suppose that $N_{5 e+1}$ is not met. So, there is a subset $X$ of $\bar{A}$ with $|\bar{A}-X|=\infty$ and $\bar{A} \leq_{m} X$ via $\varphi_{e}$. Then necessarily it has to be $r\left(5 e+1, s_{0}\right)=-1$. In fact, if it were $r\left(5 e+1, s_{0}\right)=u>-1$ for some $u$, then there is a stage $s^{\prime}+1<s_{0}$ at which the number $\varphi_{e, s^{\prime}}(u)$ has been enumerated into $A_{s^{\prime}+1}$ and $N_{5 e+1}$ was not injured up to $s_{0}$. Since by hypothesis $N_{5 e+1}$ will not be injured anymore after $s_{0}$, it would be $u \in \bar{A}$ and $\varphi_{e}(u) \notin X$, contrary to the assumption that $\varphi_{e} m$-reduces $\bar{A}$ to $X$. Let

$$
k_{5 e+1}=\lim \inf _{s \rightarrow \infty}(\max \{r(i, s): i<5 e+1\}),
$$

which is finite by Corollary 4.12. Requirements $P_{5 e}$ and $N_{5\langle i, e\rangle+2}$ are met for every $e, i \geq 0$, therefore the set $\bar{A}$ is immune. By Lemma 3.3 the set $\left\{\varphi_{e}(x): \varphi_{e}(x) \neq x \wedge x \in \bar{A}\right\}$ is infinite. By (11) there are infinitely many stages $s$ such that

$$
\max \{r(t, s): t<5 e+1\}=k_{5 e+1} .
$$

So, let $s^{\prime}+1 \geq s_{0}$ be the minimum stage such that

$$
\begin{gathered}
\max \left\{r\left(t, s^{\prime}\right): t<5 e+1\right\}=k_{5 e+1} \text {, and } \\
\text { there exists } x \leq s^{\prime}+1 \text { with } x \in \overline{A_{s^{\prime}}}, \varphi_{e, s^{\prime}}(x) \downarrow \neq x \text { and } \varphi_{e, s^{\prime}}(x)>k_{5 e+1} .
\end{gathered}
$$

Let $x^{\prime}$ be the minimum $x$ satisfying (13). This means that $N_{5 e+1}$ requires attention at stage $s^{\prime}+1$ via $x^{\prime}$, and $N_{5 e+1}$ has the highest priority by hypothesis, that is $N_{5 e+1}$ is active. At stage $s^{\prime}+1$ the number $\varphi_{e, s^{\prime}}\left(x^{\prime}\right)$ is enumerated into $A_{s^{\prime}+1}$ and $r\left(5 e+1, s^{\prime}+1\right)=x^{\prime}$. After stage $s^{\prime}+1 N_{5 e+1}$ will not be injured anymore, therefore $x^{\prime} \in \bar{A}$ and $\varphi_{e}\left(x^{\prime}\right) \notin \bar{A}$, contrary to the assumption that $\bar{A} \leq_{m} X$ via $\varphi_{e}$.

- Now, let us prove that each $N_{5\langle e, m, n\rangle+4}$ is met. Let $e, m, n$ be fixed, and let us suppose that both $\varphi_{e}^{\bigoplus_{i \in \alpha_{m}} B_{i}}$ and $\varphi_{e}^{\bigoplus_{i \in \alpha_{n}} B_{i}}$ are total and equal to the characteristic function of a set $D$. Then

$$
\lim _{s \rightarrow \infty} l(5\langle e, m, n\rangle+4, s)=\infty
$$

We have to prove that $D$ can be decided with the aid of the oracle $\bigoplus_{i \in \alpha_{m} \cap \alpha_{n}} B_{i}$. First, we fix some terminology and parameters. For every set $X$ and every natural number $n, X\lceil n$ denotes the set $X \cap\{0,1, \ldots, n\}$. If $\langle e, m, n\rangle=0$ then we pose $k=0$ and $S=\mathbb{N}$, otherwise we pose

$$
k=\lim \inf _{s \rightarrow \infty}(r(5(\langle e, m, n\rangle)-1)+4)
$$

and

$$
S=\{s: r(5(\langle e, m, n\rangle-1)+4, s)=k\} .
$$

Let $s_{0}$ be the minimum stage after which no requirement of higher priority than $N_{5\langle e, m, n\rangle+4}$ is active after $s_{0}$ and such that, by (15), for every $s \geq s_{0}$

$$
r(5(\langle e, m, n\rangle-1)+4, s) \geq k .
$$

Finally, for every $x \in \mathbb{N}$, we say that the computation $\varphi_{e, s}^{\bigoplus_{i \in \alpha_{m}} B_{i, s}}(x) \downarrow$ is $\left[\bigoplus_{i \in \alpha_{m} \cap \alpha_{n}} B_{i}\right]$-correct if

$$
\left(\bigoplus_{i \in \alpha_{m} \cap \alpha_{n}} B_{i, s}\right) \uparrow u_{\alpha_{m}}=\left(\bigoplus_{i \in \alpha_{m} \cap \alpha_{n}} B_{i}\right) \uparrow u_{\alpha_{m}},
$$

where $u_{\alpha_{m}}=u\left(\bigoplus_{i \in \alpha_{m}} B_{i, s} ; e, x, s\right)<s$.

Similarly, we define the computation $\varphi_{e, s}^{\bigoplus_{i \in \alpha_{n}}} B_{i, s}(x) \downarrow$ to be $\left[\bigoplus_{i \in \alpha_{m} \cap \alpha_{n}} B_{i}\right]$-correct with $\alpha_{n}$ in place of $\alpha_{m}$. An informal algorithm to decide $D$ with oracle $\bigoplus_{i \in \alpha_{m} \cap \alpha_{n}} B_{i}$ is the following: given any $y \in \mathbb{N}$, find a stage $s \geq s_{0}$ and $s \in S$ such that:

(i) $l(\langle e, m, n\rangle, s)>y$,

(ii) $s$ is $\langle e, m, n\rangle$-expansionary,

(iii) both the computations $\varphi_{e, s}^{\bigoplus_{i \in \alpha_{m}} B_{i, s}}(y)$ and $\varphi_{e, s}^{\bigoplus_{i \in \alpha_{n}} B_{i, s}}(y)$ are $\left[\bigoplus_{i \in \alpha_{m} \cap \alpha_{n}} B_{i}\right]$-correct. 
In particular, condition (iii) can be cheked with the oracle $\left[\bigoplus_{i \in \alpha_{m} \cap \alpha_{n}} B_{i}\right]$ for every $y \geq y_{0}$, where $y_{0}$ depends on the fixed elements $e, m, n, s_{0}$. Then,

$$
y \in D \Leftrightarrow \varphi_{e, s}^{\bigoplus_{i \in \alpha_{n}} B_{i, s}}(y)=1 .
$$

The correctness of the algorithm follows from the following lemma, which is the Tomasons' method, whose proof here is simpler than Thomason's proof, because we did not need followers in our construction.

Lemma 4.14 Given any $y \in \mathbb{N}$, let $s \geq s_{0}$ be any $\langle e, m, n\rangle$-expansionary stage in $S$ such that (i), (ii) and (iii) above are true, in particular let $z \in \mathbb{N}$ such that

$$
\varphi_{e, s}^{\bigoplus_{i \in \alpha_{m}} B_{i, s}}(y)=\varphi_{e, s}^{\bigoplus_{i \in \alpha_{n}} B_{i, s}}(y)=z .
$$

Then, for every stage $t \geq s$, at least one of either
a) $\varphi_{e, t}^{\bigoplus_{i \in \alpha_{m}}} B_{i, t}(y)=z$ or
b) $\varphi_{e, t}^{\bigoplus_{i \in \alpha_{n}}} B_{i, t}(y)=z$

is true via an $\left[\bigoplus_{i \in \alpha_{m} \cap \alpha_{n}} B_{i}\right]$-correct computation.

Proof Let $\left\{s_{j}\right\}_{j \geq 1}$ be the sequence of the infinitely many $\langle e, m, n\rangle$-expansionary stages in $S$ from $s$ on, that is

$$
s=s_{1}<s_{2}<\cdots .
$$

We prove by induction on the index $j$ that

$$
\bigoplus_{e, s_{j}} \bigoplus_{i \alpha_{m}} B_{i, s_{j}}(y)=\varphi_{e, s_{j}} \bigoplus_{i \alpha_{n}} B_{i, s_{j}}(y)=z
$$

and that for each $j \geq 1$ and for every $t$ with $s_{1} \leq t \leq s_{j}$ at least one of either a) or b) is true. For $j=1,(22)$ is true, thus both a) and b) hold. For $j \geq 1$, let us suppose that the statement is true for the $\langle e, m, n\rangle$-expansionary stage $s_{j} \in S$, that is both a) and b) hold for $t=s_{j}$, with either a) or b) true for every $t$ with $s_{1} \leq t \leq s_{j}$, and let us prove the statement for the $\langle e, m, n\rangle$-expansionary stage $s_{j+1} \in S$. Let us pose

$$
u_{\alpha_{m}}=u\left(\bigoplus_{i \in \alpha_{m}} B_{i, s_{j}} ; e, x, s_{j}\right)
$$

and

$$
u_{\alpha_{n}}=u\left(\bigoplus_{i \in \alpha_{n}} B_{i, s_{j}} ; e, x, s_{j}\right)
$$

At the stage $s_{j}+1$ at most one element $q$ is removed from $\left(\bigoplus_{i \in \alpha_{m}} B_{i, s_{j}}\right) \cup\left(\bigoplus_{i \in \alpha_{n}} B_{i, s_{j}}\right)$. But at most one computation of either a) or b) is destroyed, because by hypothesis these computations are both $\left[\bigoplus_{i \in \alpha_{m} \cap \alpha_{n}} B_{i}\right]$-correct: if both the computations a) and b) were destroyed, then it means that element $q$ would be removed from both the oracles

$$
\bigoplus_{i \in \alpha_{m}} B_{i, s_{j}}
$$

and

$$
\bigoplus_{i \in \alpha_{n}} B_{i, s_{j}}
$$

that is $q$ would be removed from $\bigoplus_{i \in \alpha_{m} \cap \alpha_{n}} B_{i, s_{j}}$, which is impossible, because

$$
\left(\bigoplus_{i \in \alpha_{m} \cap \alpha_{n}} B_{i, s_{j}}\right)\left\lceil\tilde{u}=\left(\bigoplus_{i \in \alpha_{m} \cap \alpha_{n}} B_{i}\right)\lceil\tilde{u},\right.
$$

where $\tilde{u}=\min \left\{u_{\alpha_{m}}, u_{\alpha_{n}}\right\}$. Without loss of generality let us suppose that the destroyed computation is b). It follows that for every $t$ with $s_{j}<t<s_{j+1}$ and $t \notin S$ it holds that

$$
r(5(\langle e, m, n\rangle)-1)+4, t)>k,
$$

so for every $t$ with $s_{j}<t<s_{j+1}$ is

$$
r(5\langle e, m, n\rangle+4, t) \geq s_{j}
$$


by the conditions (2) and (3) of the Definition 4.10 of the restraint function. By hypothesis no requirement of higher priority of $N_{5\langle e, m, n\rangle+4}$ is active after $s_{0}$. But $s_{0}<s_{j}$, hence for every $t$ with $s_{j} \leq t \leq s_{j+1}$, is

$$
\left(\bigoplus_{i \in \alpha_{m}} B_{i, t}\right)\left\lceil s_{j}=\left(\bigoplus_{i \in \alpha_{m}} B_{i, s_{j}}\right)\left\lceil s_{j},\right.\right.
$$

therefore for the computation a) it holds that for every $t$ with $s_{j} \leq t \leq s_{j+1}$

$$
\varphi_{e, t}^{\bigoplus_{i \in \alpha_{m}} B_{i, t}}(y)=\varphi_{e, s_{j}}^{\bigoplus_{i \in \alpha_{m}} B_{i, s_{j}}}(y)=z
$$

Now, stage $s_{j+1}$ is $\langle e, m, n\rangle$-expansionary, therefore for $t=s_{j+1}$ both the computations a) and b) will again be equal to $z$ and both $\left[\bigoplus_{i \in \alpha_{m} \cap \alpha_{n}} B_{i}\right]$-correct.

This concludes the proof of the Lemma 4.14.

This concludes the proof that each requirement is met.

This concludes the proof of the theorem.

Corollary 4.15 The countable atomless Boolean algebra is lattice embeddable into $\left(\left(I_{m}-C\right) \cup\{\mathbf{0}\}, \leq\right)$.

Proof It is known that the countable atomless Boolean algebra of subsets of $\mathbb{N}$ is uniformly recursive (see for example (Vermeeren, 2010)), so the statement follows from Theorem 4.2.

Corollary 4.16 Every countable distributive lattice is lattice embeddable into $\left(\left(I_{m}-C\right) \cup\{\mathbf{0}\}, \leq\right)$.

Proof In (Vermeeren, 2010) it has been proved that every countable distributive lattice is lattice embeddable into the countable atomless Boolean algebra. So, the statement follows from Corollary 4.15.

Corollary 4.17 Every countable partial ordering is embeddable into $\left(I_{m}-C, \leq\right)$.

Proof We observe that Theorem 4.2 applied to the uniformly recursive Boolean algebra $\mathcal{F}$ of all the finite co-finite subsets of $\mathbb{N}$ produces a recursively independent collection $\left\{B_{i}\right\}_{i \in \mathbb{N}}$, that is for every $i \in \mathbb{N}$,

$$
B_{i} \varliminf_{T} \bigoplus_{j \neq i} B_{j} .
$$

In fact, by Claim 4.5 the condition (a) in the proof of Theorem 4.2 is true, and this implies that

$$
\left(\bigoplus_{j \in\{i\}} B_{j}\right) \mathbb{Z}_{T}\left(\bigoplus_{j \in \mathbb{N}-\{i\}} B_{j}\right),
$$

is true for every $\{i\}$ and $\mathbb{N}-\{i\}$ of $\mathcal{F}$, which implies (25). So, we have the following:

- the existence of a recursively independent set $\left\{B_{i}\right\}_{i \in \mathbb{N}}$ with $\bigoplus_{i \in \alpha} B_{i}$ co-r.e., $m$-introimmune and low ${ }_{1}$ for every nonempty recursive set $\alpha \subseteq \mathbb{N}$, and

- the known fact that every countable partial ordering is embeddable in an opportune recursive partial ordering $(\mathbb{N}, \leq)$ (Mostowski, 1938).

Let $\left(P, \leq_{P}\right)$ be a countable partial ordering, and let $g: P \rightarrow \mathbb{N}$ be an embedding of $\left(P, \leq_{P}\right)$ into $(\mathbb{N}, \leq)$. For every $a \in \mathbb{N}$, let us define the nonempty recursive set $\alpha_{a}=\{x \in \mathbb{N}: x \leq a\}$. The map $f: \mathbb{N} \rightarrow I_{m}-C$ defined as $f(a)=\operatorname{deg}\left(\bigoplus_{i \in \alpha_{a}} B_{i}\right)$ is an embedding of $(\mathbb{N}, \leq)$ into $\left(I_{m}-C, \leq\right)$, becasue it is one-one and $a \leq b \Leftrightarrow \alpha_{a} \subseteq \alpha_{b}$. So, the map $f \circ g$ is an embedding of $\left(P, \leq_{P}\right)$ into $\left(I_{m}-C, \leq\right)$. 


\section{Final Observation}

We do not known if $\left(I_{m}, \leq\right)$ is an upper semi-lattice; however, we observe here that if it is, then it cannot be proved by the direct sum of the representatives, in the sense that in general it is not true that if $X$ and $Y$ are both $m$-introimmune, then $X \oplus Y$ is $m$-introimmune. We give an example of two sets $X$ and $Y$ which are both $m$-introimmune and Turing incomparable, while their direct sum is not $m$-introimmune. Let us take the three infinite sets $B_{0}, B_{1}$ and $B_{2}$ from the collection $\left\{B_{i}\right\}_{i \in \mathbb{N}}$ of the proof of Corollary 4.17. Let us consider the two sets

$$
X=\bigoplus_{i \in\{0,2\}} B_{i}
$$

and

$$
Y=\bigoplus_{i \in\{1,2\}} B_{i}
$$

Both $X$ and $Y$ are $m$-introimmune and Turing incomparable. Then, $X \oplus Y$ is not $m$-introimmune, because $X \oplus Y \leq_{m}$ $(X \oplus Y)-\left\{\langle\langle x, 2\rangle, 0\rangle: x \in B_{2}\right\}$ via the recursive function $f(\langle\langle x, 2\rangle, 0\rangle)=\langle\langle x, 2\rangle, 1\rangle$ and $f(u)=u$ for every $u \neq\langle\langle x, 2\rangle, 0\rangle$ for each $x$. In this example, however, $\operatorname{deg}(X \oplus Y)$ is $m$-introimmune, because $X \oplus Y$ is Turing equivalent to $\bigoplus_{i \in\{0,1,2\}} B_{i}$, and the latter is $m$-introimmune.

\section{References}

Ambos-Spies, K (2003). Problems which cannot be reduced to any proper subproblems. In: Proc. 28th International Symposium MFCS 2003, Lectures Notes in Computer Science (B. Rovan and P. Vojtáš, eds.)(2747), 162-168. Springer, Berlin.

Cintioli, P. (2005). Sets without subsets of higher many-one degrees. Notre Dame J. Form. Log., 46(2), $207-216$. http://dx.doi.org/10.1305/ndjfl/1117755150

Cintioli, P. (2011). Low sets without subsets of higher many one degrees. Math. Log. Quart., 57(5), 517-523. http://dx.doi.org/10.1002/malq.200920043

Cintioli, P. (2014). A minimal pair of Turing degrees. Journal of Mathematics Research, 6(1), 76-84. http://dx.doi.org/10.5539/jmr.v6n1p76

Cintioli, P., \& Silvestri R. (2003). Polynomial time introreducibility. Theory Comput. Syst., 36(1), 1-15. http://dx.doi.org/10.1007/s00224-002-1040-z

Jockusch, Jr. C. G. (1973). Upward closure and cohesive degrees. Israel J. Math., 15, 332-335. http://dx.doi.org/10.1007/BF02787575

Odifreddi, P. (1981). Strong reducibilities. Bull. Amer. Math. Soc.(N.S), 4(1), 37-86. http://projecteuclid.org/euclid.bams/1183547849

Odifreddi, P. (1989). Classical Recursion Theory I. Studies in Logic and the Foundations of Mathematics, 125, NorthHolland, Amsterdam.

Odifreddi, P. (1999). Classical Recursion Theory II. Studies in Logic and the Foundations of Mathematics, 143, NorthHolland, Amsterdam.

Simpson, S. G. (1978). Sets which do not have subsets of every higher degree. J. Symb. Log., 431, 135-138. http://dx.doi.org/10.2307/2271956

Soare, R. I. (1969). Sets with no subset of higher degree. J. Symb. Log., 34(1), 53-56. http://dx.doi.org/10.2307/2270981

Soare, R. I. (1987). Recursively enumerable sets and degrees, Perspectives in Mathematical Logic. Springer-Verlag.

Thomason, S. K. (1971). Sublattices of the recursively enumerable degrees. Z. Math. Logik Grundlag. Math., 17, 273-280. http://dx.doi.org/10.1002/malq.19710170131

Vermeeren, S. (2010). Embeddings into the countable atomless Boolean algebra. arXiv:1006.4479v1

\section{Copyrights}

Copyright for this article is retained by the author(s), with first publication rights granted to the journal.

This is an open-access article distributed under the terms and conditions of the Creative Commons Attribution license (http://creativecommons.org/licenses/by/3.0/). 